\title{
ANÁLISIS PSICOSOCIAL DEL MALTRATO INFANTIL
}

\section{Psychosocial analysis of child abuse}

Jenny Elsa Junco Supa*

\section{Resumen}

El presente artículo presenta una revisión bibliográfica y de investigaciones respecto a la prevalencia, características, consecuencias y los factores de riesgo asociados de la violencia hacia los niños, niñas y adolescentes en los espacios privados y públicos, asimismo desarrolla y explica las formas de maltrato infantil que se derivan de otros problemas sociales en la cual está involucrado no sólo los cuidadores directos sino el Estado. Se plantea el estudio de esta problemática desde la teoría de las necesidades y de los factores de riesgo, se involucra en la prevención de este problema social a la familia, escuela y comunidad.

Palabras Clave: maltrato infantil, factores de riesgo, estereotipos, variables psicosociales.

\begin{abstract}
This article presents a bibliographical and research review about the prevalence, characteristics, consequences and risk factors associated with violence against children and adolescents in private and public spaces. This study also develops and explains the forms of child abuse derived from other social problems in which direct caregivers and the State are involved. This problem is studied based on the theory of needs and risk factors. Furthermore, the family, school and community are involved in the prevention of this social problem.
\end{abstract}

Keywords: child abuse, risk factors, stereotypes.

\footnotetext{
* Psicóloga. Docente de la Universidad Femenina del Sagrado Corazón. jennyjunco@hotmail.com, jennypsicología@hotmail.com
} 


\section{INTRODUCCIÓN}

Quisiera empezar escribiendo una frase alentadora con relación al buen trato humano que reciben los niños, niñas y adolescentes del mundo. Intento evocar las frases positivas y reivindicadoras ante una sociedad indiferente a las necesidades de los niños, pero, me detengo al darme cuenta que la humanidad está cada vez más recrudecida en mostrar su indolencia e indiferencia contra aquellos seres humanos más vulnerables por su condición biológica, psíquica y maduracional que son nuestros niños y adolescentes.

Hubo un hecho que motivó seguir investigando acerca de esta problemática, el caso Pierina, una niña de nueve años que fue maltratada sistemáticamente por su progenitora y que un 11 de noviembre de 2011, después de maltratarla físicamente e incluso sexualmente la asfixió hasta causarle la muerte. Este caso que generó impacto legal ya que la madre fue condenada a cadena perpetua, debe ser de interés de la ciudadanía y del Estado no sólo para sensibilizarnos sino para asumir un activismo en la prevención e intervención oportuna.

Según datos informativos de UNICEF a diario miles de niños y niñas en el Perú son maltratados física y psicológicamente por sus padres, madres, parientes, profesores o por cualquier adulto que considera al castigo físico como normal, natural, aceptable e incluso hasta "necesario". Muchas de estas víctimas son participes de la cifra oculta que no son registradas como datos estadísticos y que se visibilizan cuando las consecuencias del maltrato son graves, como lesiones que originan incapacidades o puede llegar a ocasionar la muerte.

Según la investigación de los registros del Instituto Nacional de Salud del Niño (INSN) desde enero de 2006 hasta septiembre de 2011, realizada por Escalante- Romero y col. señalan que de 1,798 registros sobre maltrato infantil el 63,9\% eran niñas y el 39,9\% fueron adolescentes, indicando además que el $60,6 \%$ de los agresores fueron varones y el $65,8 \%$ de las agresiones ocurrieron en casa, asimismo el 48,6\% fueron registros de agresión sexual, siendo más frecuente en niñas $(73,2 \%)$ y adolescentes $(44,4 \%)$; en el 9,6\% de los casos existió coito.
Estos datos son alarmantes, puesto que la casa es el lugar más inseguro y de riesgo para los niños y adolescentes donde habita el agresor o agresora y puede con facilidad ejercer cualquier tipo de maltrato siendo el más frecuente el abuso sexual, ejerciendo el abuso de poder y su investidura de adulto sobre el menor.

La Fundación Ayuda a Niños y Adolescentes en Riesgo (ANAR) han registrado 894 casos de niñas y niños de 6 a 11 años y adolescentes varones y mujeres de 12 a 17 años que han utilizado el Teléfono ANAR, para dar a conocer que son víctimas de algún tipo de maltrato infantil durante el año 2005. Estos datos son significativos por que las víctimas directas son las que han hecho conocer que han sido y son maltratados expresando así sus voces de auxilio y que necesitan ayuda considerando además que muchas ellos son renuentes adenunciarporquese encuentran intimidados, amenazados y maniatados psicológicamente.

En un país como el nuestro bilingüe y multicultural, una población invisibilizada son los niños y adolescentes quechuahablantes, según (ANAR 2014), los niños de Cusco, Ayacucho y Apurímac son los más maltratados físicamente, principalmente por la madre, agresiones que van desde jalones de orejas hasta golpes con un palo.

Según el análisis de la Encuestas Demográfica y Salud Familiar (ENDES 2000), la madre adolescente es fuente de maltrato infantil, el $61 \%$ de las muertes infantiles en menores de cinco años es ocasionada por los padres, $30 \%$ de estas muertes son ocasionadas por la madre, el abandono del padre que deja el hogar exclusivamente a cargo de la madre, constituye otra manera de maltrato infantil, muchas de estas madres adolescentes han asumido una maternidad a temprana edad como consecuencia de haber sido víctimas de abuso sexual, tenemos así que durante el año 2010, el tipo de violencia que se ha reportado con mayor frecuencia en adolescentes ha sido el abuso sexual, llegando a 2,126 casos de los cuales 237 resultaron embarazadas incluyendo 2 niñas de 11 años, datos proporcionados por El Programa Nacional Contra la Violencia Familiar y Sexual a través de los Centros de Emergencia Mujer (CEM 2010). 
Esta situación de la madre adolescente no solo es un problema social sino que es una situación que desencadena problemas de salud pública.

Ante un historial de casos visibilizados podemos señalar que, el maltrato de menores se refiere a cualquier comportamiento de los padres, cuidadores, otros adultos o adolescentes mayores que están fuera de las normas de conducta y conllevan un riesgo importante de causar daño físico o emocional a un niño o joven. Tales comportamientos pueden ser intencionales o no intencionales, y pueden incluir actos de omisión (es decir, el abandono) y comisiones (vale decir, el abuso) (Bromfield, 2005; Cristofel et al, 1992; Gilbert et al, 2009).

Escuchar las voces de los niños, revisando las historias de muchos que, con su silencio, vivían una tortura intensa y prolongada, permiten conocer que las formas de maltrato convergen unas con otras, es así que, las secuelas desarrollan un trauma complejo que refleja los múltiples síntomas que interactúan, trastornos, múltiples experiencias adversas y la amplia gama de resultados cognitivos, afectivos y conductuales asociados con el trauma prolongado, especialmente si dicho trauma se producen a temprana edad y que implican un elemento interpersonal (por ejemplo, el abuso sexual) (Precio -Robertson, Rush, Wall, y Higgins, 2013). El trauma complejo afecta el desarrollo del cerebro y puede interferir con la capacidad del niño para integrar la información sensorial, emocional y cognitivo, que puede conducir a efectos posteriores tales como problemas de salud cognitiva, conductual, física y mental (Consejo Nacional de la Ciencia en el desarrollo Infantil [NSCDC], 2007; Perry, 2001; Streeck-Fischer \& vander Kolk, 2000).

\section{REPRESENTACIONES SOCIALES ACERCA DEL MALTRATO}

Las representaciones sociales basadas en mitos y creencias que tienen los adultos, padres, cuidadores, profesores incluso aquellos que tienen la obligación tuitiva de atender y proteger al menor, naturalizan e invisibilizan las formas de maltrato infantil. $\mathrm{Al}$ respecto la Defensoría del Pueblo del Perú de la Adjuntía para la niñez y la adolescencia (2009), señala estos mitos y creencias vigentes, consideradas como vallas que limitan y obstaculizan salvaguardar los derechos de los niños y adolescentes.

\section{a. Los niños y niñas nacen $y$ crecen en una sociedad "adultista".}

En la antigüedad, el niño era considerado como un proyecto de persona adulta, caracterizado por su irracionalidad e incapacidad, al cual se le trataba como un objeto y propiedad del adulto, Este esquema que se mantiene influye en la manera de disciplinarlos y corregirlos utilizando la violencia. Es así que se ha mantenido en la sociedad la cultura "adultista" o "adultocéntrica", donde el niño no sólo es considerado como incapaz, sino invisible o minimizado en el espacio social. Doctrina que fortalece el desencuentro intergeneracional.

En la actualidad esta brecha intergeneracional ha ido variando en la medida que se han dado cambios legislativos y nuevos enfoques contemporáneos de desarrollo de los niños y adolescentes, se le involucra en el dinamismo de la familia pero al mismo tiempo esta familia está alejada e indiferente a las necesidades de los menores.

\section{b. La "naturalización" del castigo y la} violencia.

Se utiliza las diferentes formas de maltrato (castigo físico, psicológico) para corregir las conductas inapropiadas de los niños y adolescentes, método de enseñanza y disciplina, el castigo físico equivocadamente ha sido visto como natural, socialmente permitido, tolerado.

El ilustre pensador Rousseau, señala, por primera vez, la existencia de las necesidades infantiles no solo desde el punto de vista físico sino psicológico a fin de que los padres puedan crear vínculos afectivos con sus hijos, esta concepción influye en el ámbito jurídico, en el siglo XIX se empezó a desarrollar la idea de que el Estado debía proteger a los niños dentro de los límites permitidos por la familia, en el siglo XX se reestructura el estudio de la niñez basado en su derechos, con la Declaración de los Derechos del Niño en 1924 y la adopción de la Convención sobre los Derechos del Niño de 1989, es así que, surge la doctrina de la protección integral en la cual el niño es atendido como sujeto de derechos. Los tratos humillantes, el castigo 
físico y otras formas de maltrato a los niños surgen como interés de las comunidades para otorgar medidas de protección, sin embargo, subsiste la tendencia de asociar la obediencia con tutela y protección, como raíces de una cultura paternalista. María Elena Mannarelli en "La infancia y la configuración de los vínculos en el Perú: un enfoque histórico" menciona: “(...) reflexionar sobre la infancia en el Perú hoy exige incluir el problema de patriarcado/paternalismo y su contraparte política, la cultura patrimonial y clientelista".

El Comercio (2009), señala que un $42 \%$ de los entrevistados dice estar de acuerdo con que algunos niños son tan desobedientes que necesitan de vez en cuando que sus padres los repriman físicamente. "Muchos peruanos siguen creyendo que es normal castigar a los hijos. También hay quienes creen que tienen el supuesto poder de castigar físicamente a los niños porque los atienden en la escuela o en otros espacios".

\section{c. La "cultura de la propiedad".}

La creencia irracional de propiedad y posesión que suelen tener los adultos con relación a sus propios hijos o aquéllos que han sido confiados se consideran legitimados de disponer de su mente, cuerpo, fuerza. Un ejemplo de esta situación se produce cuando el padre autoriza al profesor de su hijo a que utilice instrumentos para disciplinarlo o corregirlo, o cuando dice a sus hijos frases como: "debes hacer lo que yo digo porque eres mi hijo". Esta "cultura de la propiedad" impregna actitudes relacionadas con el castigo bajo el disfraz de buscar lo mejor para los niños o su bienestar.

\section{d. Una sociedad "patriarcal".}

Una forma en la cual se ha estructurado las relaciones sociales, es la dominación del hombre adulto sobre la mujer y el niño, una división de género, generacional y de organización de poder, cultura patriarcal en la cual se ha naturalizado el comportamiento de maltrato no solo por el hombre sino también por la mujer en agravio de los niños.

En consonancia con los trabajos de Vega y Moro (2013), se halló que la representación social del maltrato intrafamiliar está condicionada por: 1) los estereotipos de género, los hombres y mujeres tienen una percepción distinta del maltrato; 2) las diferencias generacionales, los jóvenes consideran como maltrato conductas que culturalmente antes no se consideraban (por ejemplo, no mostrar afecto); y, 3) la experiencia personal de haberse sentido maltratado de pequeños genera creencias y percepciones diferentes de la realidad.

En consecuencia, los adultos tienen diferentes representaciones sociales con relación a las formas de maltrato, gravedad y frecuencia, basada en sus experiencias y percepciones.

La travesía que viven miles de niños y adolescentes en el mundo, en sociedades donde la violencia es el instrumento de poder, donde persiste la indiferencia y en donde se vulnera sus Derechos de Niño y Adolescente trae como consecuencias efectos psicosociales graves y de impacto que en muchos casos son irreversibles.

\section{EFECTOS PSICOSOCIALES DEL MALTRATO INFANTIL:}

\section{I.- Efectos en el Desarrollo Integral del Niño, Niña y Adolescente: \\ Consecuencias para la salud física}

Lesiones abdominales o toráxicas.

Lesiones cerebrales.

Moretones e hinchazón.

Quemaduras y escaldaduras.

Lesiones del sistema nervioso central.

Fracturas.

Desgarros y abrasiones.

Lesiones oculares.

Discapacidad irreversible y/o transitoria.

Consecuencias sexuales y reproductivas

Problemas de salud reproductiva.

Disfunción sexual.

Enfermedades de transmisión sexual, como la infección por el VIH y el SIDA.

Embarazos no deseados.

\section{Consecuencias psicológicas}

Abuso de alcohol y otras drogas.

Disminución de la capacidad cognoscitiva.

Comportamientos delictivos, violentos y de otros tipos que implican riesgos.

Depresión y ansiedad. 
Retraso del desarrollo.

Trastornos de la alimentación y el sueño.

Sentimientos de vergüenza y culpa.

Hiperactividad.

Incapacidad para relacionarse.

Desempeño escolar deficiente.

Falta de autoestima.

Trastorno postraumático por estrés.

Trastornos psicosomáticos.

Comportamiento suicida y daño autoinfligido.

Cambios en su sistema de valores.

Desconfianza (entorno social).

Cambios en su personalidad (irritabilidad, dependencia etc.).

\section{Otras consecuencias de salud a largo plazo}

Cáncer.

Enfermedad pulmonar crónica.

Síndrome de colon irritable.

Cardiopatía isquémica.

Enfermedad hepática.

Salud Psicológica.

Problemas de salud reproductiva, como la esterilidad.

\section{Consecuencias económicas}

Costos directos: Tratamiento, visitas al médico de hospital y otros servicios de salud.

Costos indirectos: Productividad, discapacidad, menor calidad de vida y muerte prematura.

Costos para el sistema sistema de justicia penal y otras instituciones:

Gastos relacionados con detener y procesar a infractores. Costos para organizaciones de bienestar social, costos asociados con hogares sustitutos, para el sistema educativo y costos para el sector de empleo que resultan del ausentismo y la baja productividad.

Fuente: Runyan D. y otros (2002). Maltrato y descuido de los menores por los padres u otras personas a cargo. En: Krug E.G. y otros (Eds).

Informe Mundial sobre la Violencia y la Salud. Washington DC, Organización Panamericana de la Salud, págs. 63-9.
Las secuelas y consecuencias como producto del maltrato a los niños, niñas y adolescentes se producen en el entorno que aparentemente son seguros y, por el contrario, son escenarios de violencia extrema como la familia, la escuela, los sistemas de protección y de justicia, el lugar de trabajo y la comunidad.

Puga (2008), en su investigación, relaciones interpersonales en un grupo de niños que reciben castigo físico y emocional señala que estos niños tienen dificultades para involucrarse en relaciones de respeto mutuo, niveles elevados de agresión, sentimientos disfóricos y alteraciones en las capacidades cognitivas.

Blumberg, H. (2011) señala la relación que existe entre abuso en la niñez y cambio en el cerebro adolescentes:

"(...) investigadores de la facultad de Medicina de la Universidad de Yale hallaron que los adolescentes que habían sido víctimas de abuso o negligencia tenían menos "materia gris", el tejido que contiene neuronas, en algunas áreas del cerebro, en comparación con los que no habían sufrido maltrato (...). Las áreas afectadas del cerebro podrían diferir entre chicos y chicas y podrían depender de si los adolescentes fueron expuestos a abuso o negligencia o si la negligencia fue física o emocional, anotaron los investigadores (...). Se observaron reducciones en la materia gris de las áreas prefrontales del cerebro, independientemente de si el adolescente había sufrido abuso físico o negligencia emocional. Pero las reducciones en la materia gris de otras áreas del cerebro dependían del tipo de maltrato. Por ejemplo, la negligencia emocional se asoció con menos materia gris en áreas del cerebro que regulan la emoción."

También debemos indicar lo señalado por Bruce, $\mathrm{L}$. y colaboradores (2012) en "Maltrato Infantil y trastorno de ansiedad social: Implicaciones para la severidad de los síntomas y respuestas a farmacoterapia" al precisar que:

"Todos los tipos de maltrato a excepción del abuso sexual y el abuso físico se relacionaron con una mayor severidad de los síntomas. El abuso emocional y el abandono se relacionaron 
con una mayor discapacidad y el abuso emocional, abandono emocional y el abuso físico se relacionaron con la disminución de la calidad de vida. El abuso emocional predijo significativamente el abandono (...). El maltrato emocional se vincula más fuertemente con la disfunción en el trastorno de ansiedad social, a pesar de una tendencia en la literatura de la ansiedad para centrarse en los efectos del abuso sexual y físico. Además los individuos que informan de abuso emocional eran más propensos al abandono de la farmacoterapia, pero aquellos que permanecieron en curso mostraron resultados similares a aquellos sin una historia de maltrato".

Ferreira, R.A. (2003) señala que los niños con abandono moral provienen de familias con funcionamiento inadecuados donde las reglas y límites no son bien definidos, considerados como familias extremas, con tendencia a ser rígidas, liderazgo autoritario y disciplina estricta.

\section{DERECHOS AFECTADOS.}

Los efectos adversos en la salud y en su desarrollo integral de los niños, niñas y adolescentes vulneran el principio básico y rector "el interés superior del niño". La vulneración de este principio afecta el derecho a la vida, el derecho a la integridad personal, el derecho a la igualdad y no discriminación, el derecho a la salud y a vivir en una familia libre de violencia, todos aquellos derechos de protección al niño y adolescente que regula las normas internacionales y nacionales que corresponde a cada país.

\section{LAS NUEVAS FORMAS DE MALTRATO INFANTIL}

Los cambios en las estructuras y organizaciones sociales, culturales y socioeconómicas han originado la aparición de nuevas formas de maltrato infantil que merecen la atención por las consecuencias a corto o largo plazo, estas formas pueden ser:

- Hijos víctimas de rupturas matrimoniales, los hijos que son utilizados como mensajeros para llevar información de cada uno de los progenitores en conflicto legal o psicológico.
- Hijos de falsos casos de bullying o acoso escolar, los padres hacen vivir a sus hijos enfermedades psicológicas como una depresión a causa de un falso bullying con el fin de conseguir un beneficio.

- Los hijos con carencias afectivas y abundantes regalos: generalmente son hijos de padres que están separados y no viven juntos, el padre o madre que visita al niño lo complace en extremo con la finalidad de mitigar su culpa.

- Fabricar hijos genios y/o perfectos.

- Niños excesivamente independientes.

- Niños sometidos a entrenamientos educativos agotadores.

- Acoso escolar y el maltrato entre iguales.

- Síndrome de alienación parental.

- Exposición al consumo.

A continuación se presenta un cuadro en detalle: 


\begin{tabular}{|c|c|c|}
\hline Maltrato Infantil & Hijo /Tipos & Características \\
\hline $\begin{array}{l}\text { En el contexto familiar } \\
\text { Hijos mensajeros }\end{array}$ & $\begin{array}{l}\text { Hijos mensajeros } \\
\text { Hijos perfectos } \\
\text { Hijos sobreestimulados } \\
\text { Hijos con roles prematuros } \\
\text { Hijos complacientes } \\
\text { Hijos alienados }\end{array}$ & $\begin{array}{l}\text { - Trasladan la información de un progenitor a otro. } \\
\text { (Padres separados, padres en proceso de divorcio etc.) } \\
\text { - Intolerante a la frustración, presión excesiva. } \\
\text { - Excesivamente retribuidos, expuestos a actividades no } \\
\text { acorde a su edad, expectativas de los padres. } \\
\text { - Asumen responsabilidades no acorde a su edad. } \\
\text { - Hijos dependientes, sumisos. } \\
\text { - Padre o madre que ante el hijo, indispone con relación } \\
\text { al padre o madre con el cual no vive. }\end{array}$ \\
\hline $\begin{array}{l}\text { En el contexto escolar, } \\
\text { recreativo, deportivo }\end{array}$ & Acoso escolar y variantes. & Maltrato entre iguales \\
\hline $\begin{array}{l}\text { En el contexto } \\
\text { Comunitario }\end{array}$ & $\begin{array}{l}\text { Niños paranoicos } \\
\text { Niños migrantes }\end{array}$ & $\begin{array}{l}\text { Percepción de la violencia ciudadana, inseguridad } \\
\text { ciudadana. } \\
\text { Expuestos a la discriminación. Desarraigo cultural, } \\
\text { sensible y vulnerable a la explotación sexual, explotación } \\
\text { laboral (trata de personas, trabajo forzoso). } \\
\text { Desarrollar en la etapa adulta el Síndrome de Ulises. }\end{array}$ \\
\hline Contexto social & $\begin{array}{l}\text { Niños consumistas } \\
\text { Niños mendigos } \\
\text { Niños expuestos al peligro. } \\
\text { Niños utilitarios de los } \\
\text { medios. }\end{array}$ & $\begin{array}{l}\text { Utilizados en publicidad sexista. } \\
\text { Grupo vulnerable, sensible para la persuasión del } \\
\text { consumismo, determinadas marcas diseña el estilo vida } \\
\text { de los niños y adolescentes basados en los intereses del } \\
\text { mercado. } \\
\text { La Infancia traicionada, pobre miserable que cuestiona la } \\
\text { sociedad de la abundancia. } \\
\text { Expuestos a la mendicidad para obtener el beneficios de } \\
\text { los adultos (incluso padres) } \\
\text { Consumo excesivo de la TV. Modelamiento de } \\
\text { comportamientos violentos. }\end{array}$ \\
\hline
\end{tabular}

Elaboración propia: Junco, Jenny (2014).

Las infinitas investigaciones reportan que la familia es un factor de protección y de riesgo para el desarrollo integral del niño y adolescente, ya que, en ella se presentan formas extremas de violencia, maltrato, carencia de afecto, comportamiento antisocial y/o desadaptativo entre otras.

Para visibilizar las otras formas de maltrato y de riesgo social de la infancia, nuestro foco de atención no solo debe centrarse en la familia sino en los riesgos que están presentes en el medio o entorno del menor.

En la actualidad las sociedades han generado nuevos escenarios y nuevas problemáticas en el proceso de socialización de la infancia que pueden estar inmersos en proceso de desadaptación, estrés pos trauma o exclusión social por otras causas que no es necesariamente la familia, sin embargo la familia es 
considerada como una variable mediadora ante estos factores de riego.

Los mecanismos para analizar estas diferencias son:

1. Analizar el medio en la cual está insertada la familia. Un niño está en riesgo social si pertenece a un entorno que presenta factores de riesgo como el consumo y expendio de las drogas, delincuencia juvenil entre otros.

2. Conocer cómo se produce la interacción del niño con ese entorno. Las carencias en el entorno generan impacto en su proceso de socialización que repercuten en sus características personales afectivas, cognitivas y sociales, la ausencia de espacios de recreación, de programas del adecuado uso del tiempo libre y del ocio.

3. Se han generado diferentes agentes de socialización en la infancia que afectan su desarrollo integral, como por ejemplo: el maltrato entre iguales, el bullying, el maltrato en el deporte, a través del consumo, del urbanismo y diseño de las ciudades.

La teoría de las necesidades de la infancia propuesta por López, Fuertes Zurita, López Gómez de Cádiz, Sánchez Redondo y Merino (1995), posibilita detectar y analizar las nuevas formas de riesgo social para el infante, esta teoría se basa en la taxonomía de necesidades, la misma que se fundamenta en los derechos de los niños y niñas y ello nos permite dinamizar los nuevos indicadores de los bloques de necesidades: 1) carácter físico biológico; 2) carácter cognitivo; y, 3) emocionales y sociales. Esta taxonomía nos va a permitir visualizar las necesidades no cubiertas en la infancia. El siguiente cuadro refleja todas las necesidades básicas, así como las situaciones consideradas de riesgo:

Figura I: Taxonomía de las Necesidades Básicas (Adaptación: López, y otros, 1995)

\begin{tabular}{|c|c|}
\hline NECESIDAD & RIESGO \\
\hline $\begin{array}{l}\text { Necesidades físico biológicas. } \\
\text { Alimentación. } \\
\text { Temperatura. } \\
\text { Higiene. } \\
\text { Sueño. } \\
\text { Actividad física: ejercicio y juego. } \\
\text { Protección de riesgos reales. } \\
\text { Salud. }\end{array}$ & $\begin{array}{l}\text { Desnutrición, déficit, no apropiadas a su edad. } \\
\text { Frío en la vivienda, humedad, falta de vestido y calzado. } \\
\text { Suciedad, parásitos. } \\
\text { Insuficiente, ruido ambiental, lugar inadecuado. } \\
\text { Inmovilidad corporal, ausencia de juego y espacios, inactividad. } \\
\text { Accidentes domésticos, castigos físicos, agresiones, accidentes de } \\
\text { circulación. } \\
\text { Falta de control, provocaciones de síntomas, no vacunación. }\end{array}$ \\
\hline $\begin{array}{l}\text { Necesidades cognitivas. } \\
\text { Estimulación sensorial. } \\
\text { Explotación física y social. } \\
\text { Comprensión de la física y social. }\end{array}$ & $\begin{array}{l}\text { Falta de estimulación lingüística, privación o pobreza sensorial, retraso en } \\
\text { el desarrollo orgánico. } \\
\text { No tener apoyo en la exploración, entorno pobre. } \\
\text { No escuchar, no responder, visión pesimista, anomia, mentir. }\end{array}$ \\
\hline $\begin{array}{l}\text { Necesidades Emocionales y Sociales. } \\
\text { Seguridad emocional. } \\
\text { Red de relaciones sociales. }\end{array}$ & $\begin{array}{l}\text { Rechazo, ausencia, no accesibles, no responder, no percibir. } \\
\text { Aislamiento social, imposibilidad de contactar con amigos, compañeros } \\
\text { de riesgo. }\end{array}$ \\
\hline $\begin{array}{l}\text { Participación y autonomía progresiva. } \\
\text { Curiosidad, imitación y contacto sexual. }\end{array}$ & $\begin{array}{l}\text { No ser escuchado, dependencia. } \\
\text { No escuchar, no responder, engañar, castigar, manifestaciones infantiles, } \\
\text { abuso sexual. }\end{array}$ \\
\hline $\begin{array}{l}\text { Protección de riesgos imaginarios. } \\
\text { Interacción lúdica. }\end{array}$ & $\begin{array}{l}\text { No escuchar, no responder, no tranquilizar, violencia verbal, amenazas, } \\
\text { pérdida de control. } \\
\text { No disponibilidad de tiempo, ausencia de iguales, no accesible. }\end{array}$ \\
\hline
\end{tabular}


A continuación desarrollaremos las formas de maltrato que han sido visibilizados y merece la atención inmediata:

\section{El maltrato entre iguales.}

El maltrato entre iguales ha sido definido como "un comportamiento prolongado de insulto verbal, rechazo social intimidación psicológica y/o agresividad física de unos niños hacia otros que se convierten, de esta forma, en víctimas de sus compañeros" (Olweus, 1993).

Ante esta realidad Sáenz, G. M (2010), refiere que el acoso escolar está relacionado con la presentación de sintomatología depresiva, hallazgo de una investigación a escolares de primero, segundo y tercero de secundaria de un colegio del Estado de Lima. Estos datos predicen el comportamiento suicida de la víctima ante un acoso escolar crónico.

Ameniya, Oliveros y Barrientos (2009) afirman que la violencia escolar severa se asocia significativamente con la reacción de los padres al conocer el hecho, repetición de la amenaza a pesar de comunicar las agresiones, presencia de pandilleros en el colegio, tener amigos pandilleros y poseer defecto físico.

\section{Niño y adolescentes migrantes.}

Las condiciones económicas de adversidad que viven muchas familias al no poder satisfacer sus necesidades y carecer de oportunidades, motivan que busquen otras alternativas en otros territorios lejanos de su ciudad de origen e incluso de su país. Es así que tenemos niños, niñas y adolescentes que ven cómo su familia se tiene que separar porque el padre o la madre emigran a otro país, o, también, el caso de adolescentes que emigran solos a un país europeo o migran a otra ciudad de su país con la esperanza de encontrar trabajo. En muchos casos estos niños y adolescentes son engañados con la promesa de tener una condición de vida diferente. En todos estos casos, así como en el resto de situaciones producidas por la emigración, nos encontramos ante una problemática social que genera una situación de riesgo para la infancia que la vive. La situación migratoria puede acarrear otros problemas como la explotación sexual, trabajo forzado y trata de personas entre otros.
Los datos proporcionados por el Departamento de Seguridad Nacional (DSH) de EE.UU (2014). Unos 70.000 menores han sido retenidos en lo que va del año fiscal en Estados Unidos y la mayor parte provino de El Salvador, Guatemala, Honduras y México.

Asimismo la Cancillería del Perú reporto en los últimos diez meses de este año 65 menores peruanos que ingresaron ilegalmente a Estados Unidos.

\section{La influencia de los medios de comunicación masivo o de masa (mass media) \\ Cabero y Romero (2002), definen los Mass Media} como "los mayores promotores de la información en el siglo XXI, realidad que no es desconocida por la sociedad pero que se tolera en pos del avance tecnológico y de las necesidades informativas de las comunidades" es así que las fuentes principales de información son las mayores fuentes de difusión de problemas sociales como la televisión, internet, radio y la prensa.

Los medio de televisión se han convertido en el trasmisor de pautas, dinámicas de vida, sistemas de valores, formas de resolución de conflictos, creencias basadas en prejuicios sexistas y discriminatorias así como un consumismo de productos relacionados con el éxito social y personal siendo la población adolescente más vulnerable.

Nos preocupa que los niños y adolescentes ven tres horas de televisión al día en el Perú según el último reporte del Consejo Consultivo de Radio y Televisión ( Concortv), el $72 \%$ lo hace porque lo relaja y entretiene; el $17 \%$, porque no tiene nada que hacer; y el $11 \%$, porque está solo, muchos padres no analizan los contenidos de los espacios, el 86,8\% de los niños y jóvenes peruanos mira espacios de entretenimientos todos los días.

Un reciente estudio de la Universidad de Michigan, desarrollado durante 15 años, ha llegado a la conclusión que los/as niños/as que ven programas violentos en televisión están más predispuestos a protagonizar actitudes y respuestas agresivas cuando son adultos. A su vez Petrus (2001) han denunciado la capacidad de modelling de los mass media como una de las causas de que los escolares adopten, como propios, patrones de conducta violentos y agresivos. 
Otro aspectos de interés está en relación al marketing de los juguetes infantiles, vía acción de los mass media, observándose que la cultura de los juegos, juguetes y diversiones infantiles este definida y creada por los expertos estrategas del marketing, al respecto Benjamín (1984), se refiere al niño como cazador cuando "Apenas entra en la vida y ya es un cazador. Caza los espíritus cuyos vestigios olfatea en las cosas" además señala que esta imagen de niño cazador y explorador se está perdiendo cuando vemos que los medios de comunicación y la sociedad del consumo de hoy determinan los nuevos juegos, los nuevos programas del mundo infantil.

Rodríguez (1991), señala que los juegos de hoy que forma parte de la pedagogía de la recreación se anticipan al deseo de los niños o corresponden al deseo de los adultos que dirigen mercado del consumo infantojuvenil.

También tenemos que considerar que los representantes del periodismo social, sostienen que los medios ejercen una influencia negativa cuando difunden los hechos relacionados con los niños, niñas y adolescentes de forma morbosa o cuando revelan datos o muestran imágenes que identifican a las víctimas.

Los cambios tecnológicos y la tecnificación de las relaciones humanas deben tener en cuenta el principio del interés superior del niño, considerando normas vinculantes como:

La declaración de Copenhague de 1995 sobre el desarrollo social refiere: "la sociedad debe ser sensible al desarrollo y el bienestar social de todos los sectores sociales, y, de forma particular, de la infancia que vive situaciones vulnerables"

\section{Síndrome de Alienación Parental: una forma de maltrato infantil.}

Richard Gardner define el Síndrome de Alienación Parental (SAP) como un desorden que surge principalmente en el contexto de las disputas por la guarda y custodia de los niños. Su primera manifestación es una campaña de difamación contra uno de los padres.

Aguilar (2005), lo define como un trastorno caracterizado por un conjunto de síntomas que resultan del proceso por el cual un progenitor transforma la conciencia de sus hijos, mediante distintas estrategias, con objeto de impedir, obstaculizar o destruir sus vínculos con el otro progenitor, los comportamientos y estrategias que el progenitor alienante pone en juego suelen ser sutiles.

Las consecuencias del SAP en menores son: trastornos de ansiedad, trastornos del sueño y alimentación y trastornos de conducta.

En nuestro país la tasa de divorcios se ha incrementado lo cual han originado las familias monoparentales, judicializándose mayormente el proceso de tenencia, régimen de visitas etc. Es en este proceso que los niños son considerados para cada progenitor trofeos de guerra.

\section{PERSPECTIVA DE ABORDAJE EN MALTRATO A LA INFANCIA.}

Para atender las necesidades de la infancia y analizar este problema del maltrato infantil es relevante analizar los múltiples factores causales, predisponentes, detonantes a distintos niveles. El Modelo Ecológico - Sistémico representa la interacción relacional entre los factores que se presentan en los diversos niveles.

El Nivel Macrosistémico: Está compuesto por valores culturales y/o étnicos, las creencias, los sucesos históricos que afectan a los otros niveles, se consideran las condiciones de tipo socioeconómica como: los recursos económicos, crisis económicas y el desempleo. De tipo estructural como las situaciones de organización y funcionamiento que afecta y limitan a los individuos al acceso de los recursos de asistencia. De tipo cultural como: las actitudes y valores predominantes en cada grupo social, educación de los niños, satisfacción de las necesidades, comprender el papel de cada miembro de la familia, etc.

El Nivel Exosistema: Comprende aquellas estructuras formales e informales que influyen o delimitan lo que acontece en el microsistema por ejemplo: el trabajo, la familia extensa, los grupos vecinales, aspectos que rodean al individuo y la familia los cuales afectan de manera directa en las relaciones sociales y el ámbito laboral. Por ejemplo, 
los acontecimientos laborales estresantes influyen en las adecuadas relaciones parentales ya que el estrés parental afecta el desarrollo socio afectivo de los hijos.

Nivel de Microsistema: Comprende el conjunto de relaciones entre la persona en desarrollo y ambiente próximo. Importa analizar las características de las diferentes etapas del desarrollo del niño y adolescente considerándose además todas aquellas variables de los comportamientos concretos e interacción de los miembros de la familia como:

- Interacción de ambos padres.

- Interacción de los padres con sus hijos.

- Interacción del padre o madre con cada uno de sus hijos.

- Los atributos de los padres como: la capacidad empática, tolerancia al estrés. Síntomas depresivos, alteraciones de personalidad, desajuste marital, violencia de pareja, interacción de variables temperamentales y comportamentales de los hijos.

- Incluye las variables: Como la propia crianza de los padres, la relación con los propios padres, el tipo de cuidado y atención recibida en su infancia.

- Considera relevante las relaciones fraternales.

Este modelo nos permite reconocer la naturaleza multicausal del maltrato infantil, no podría atenderse esta problemática psicosocial sin tomar en cuenta la comunidad, la cultura y otros contextos donde se inserta el individuo y la familia que recibe la influencia de todas las estructuras políticas. ecológicas, ambientales, culturales, económicas, tecnológicas etc.

Modelo ecológico de los factores de riesgo de maltrato adaptado por Palacios, Jiménez, Oliva y Saldaña (1998).

\begin{tabular}{cccc}
\hline DESARROLLO & MICROSISTEMA & EXOSISTEMA & MACROSISTEMA \\
ONTOGENÉTICO &
\end{tabular}

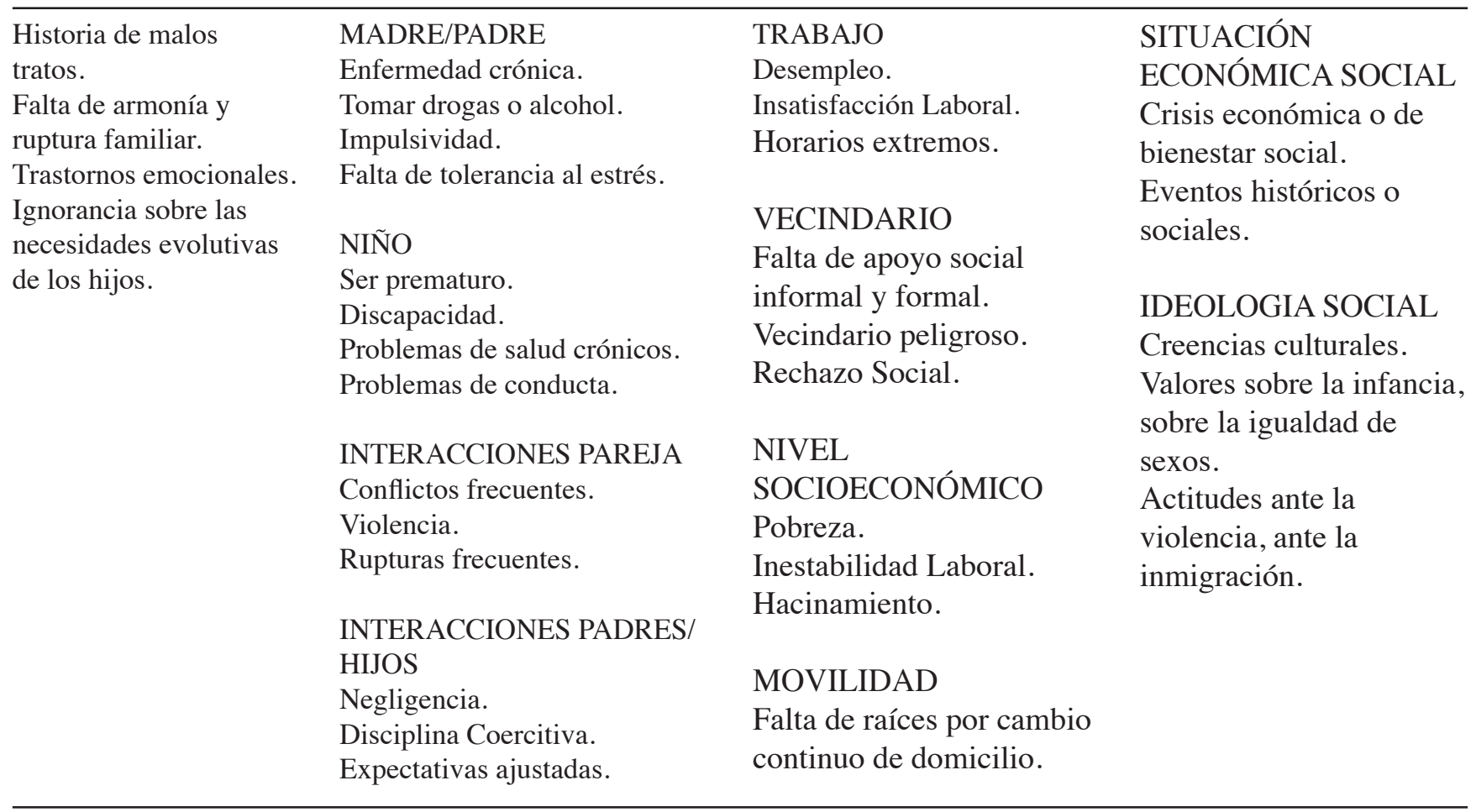


En conclusión, a partir de este enfoque se pueden identificar los presupuestos de intervención que se debe considerar en el diseño de programas de intervención, prevención del maltrato a la infancia y adolescencia así como el planteamiento de las políticas públicas.

\section{REFLEXIONES:}

- Estar conscientes de nuestra sensibilidad ante esta problemática. Ser empáticos para atender las verdaderas necesidades del niño, niña y adolescentes.

- Procurar que los menores crezcan y se desarrollen en una familia, a pesar que la familia es un factor de riesgo, que naturaliza el castigo como forma de corrección.

- La diversas formas violentas de maltrato ocasionan secuelas devastadoras en la psicología del ser humano.

- Se valida el modelo trangeneracional en la cual el padre o madre progenitora que ha sido maltratada en su infancia tendera a maltratar a sus hijos

- Las nuevas formas de maltrato está en relación con la globalización a través del uso de los medios de comunicación masiva.

- La labor preventiva en la cual se involucre a la familia, escuela y comunidad será una vía alterna de poder reducir la tasa de maltrato.

\section{REFERENCIAS}

Adjuntía para la niñez y la adolescencia de la Defensoria del Pueblo. (2009). Adiós al Castigo. Lima.

Andrade, J. A., Céspedes, K. \& Villamil, E. (2012). Medios de Comunicación y Comportamientos agresivos en los adolescentes. Revista PsicologiaCientifica.com, 14(25). Recuperado de: http://www.psicologiacientifica.com/mediosde-comunicacion-comportamientos-agresivosadolescentes.
Ayerbe, P. (2000). Convivencia y violencia en la ESO: aspectos didácticos organizativos, en Lorenzo, M.; García, M.D.; Torres, J.A.; Ortega, J.A.; Debón, S. y Notoria, A. (eds.) Las organizaciones educativas en la sociedad neoliberal. Granada, Grupo editorial universitario, 339-368.

Balsells, A. (1997). Maltractament infantil i educación familiar. Lleida: Universitat de Lleida.

Blumberg, H. (2011). El abuso en la niñez podría asociarse con cambios en el cerebro adolescente. Psiquiatría.com. Archives of Pediatric and Adolescent Medicine.

Bruce, L., Heimberg, R., Blanco, C., Schneider, F., Liebowitz, M. (2012). Maltrato Infantil y trastorno de ansiedad social: Implicaciones para la severidad de los síntomas y la respuesta a la farmacoterapia. Articulo Depresión y Ansiedad. Feb; 29 (2):132139. Recuperado de htpp//www.psiquiatria.com/ artículos/ansiedad/fobia social/56254/

De Paul Ochotorena, J. y Arruabarrena, M.I (1996). Manual de protección infantil. Barcelona: Masón.

Díaz-Aguado, M.J. (1999). Programas de educación para la tolerancia y prevención de la violencia en los jóvenes. Volumen 1. Fundamentación psicopedagógica. Madrid: Ministerio de trabajo y asuntos sociales.

Escalante, L., Huamaní, Ch., Serpa, H., Urbano. Durand, C., Farfán, M., Ferrer, C., Granados, G. (2012). Maltrato infantil y del adolescente registrado en un hospital de referencia nacional, 2006 - 2011. Rev. Perú. med. exp. salud pública [online].29 (1), pp. 28-34. Recuperado de http:// www.scielo.org.pe/pdf/rins/v29n1/a05v29n1

Ferreira, R. A. (2003). Sistema de interacción familiar asociado a la autoestima en menores en situación de abandono moral o prostitución. Lima: Universidad Nacional Mayor de San Marcos.

Fundación ANAR (2005). Estudio descriptivo del maltrato infantil una experiencia desde el teléfono Lima: ANAR.

Fundación ANAR (2014). Sistematización del Teléfono ANAR en Quechua. Lima.

Gracia, E. \& Musitu, G. (1993). El maltrato infantil. Un análisis ecológico de los factores de riesgo. Madrid: Centro de Publicaciones del Ministerio de Asuntos Sociales. 
Gómez De Terreros, I. (1995). Los profesionales de la salud ante el maltrato infantil. Granada: Comares.

Jara, C. L, (2014). Representaciones sobre el maltrato en niños limeños y andinos a través de sus dibujos. Lima: Fondo Editorial de la Asamblea Nacional de Rectores.

López, F.; López, B.; Fuertes, J. Sánchez, J.M. y Merino, J. (1995): Necesidades de la infancia y protección infantil. Madrid, Ministerio de Asuntos Sociales.

Organización Mundial de la Salud (OMS) (2002). Resumen Informe Mundial sobre la violencia y de la Salud. Washington, DC.: Organización Panamericana de la Salud para la Organización Mundial de la Salud.

Organización Mundial de la Salud (OMS) (2009). Prevención del Maltrato Infantil: Qué hacer y cómo obtener evidencias. Washington D.C.: OMS

Petrus, A. (1997) Nuevas formas de maltrato infantil. En Balsells, A., Maltractament infantil $i$ educació familiar. Lleida, Universitat de Lleida., 9-45. Recuperado de:http://campus.usal. es/ teoriaeducacion/rev_numero_04/n4_art_ balsells.htm.
Petrus, A. (2001). Cultura de la violencia y educación secundaria. Revista española de educación comparada. 7. 23-51.

Pinheiro, P. S. (2006). Acabar contra la violencia de niños, niñas y adolescentes. Naciones Unidas.

Puga, V. L. (2008). Relaciones interpersonales en un grupo de niños que reciben castigo físico $y$ emocional. Tesis para optar por el título de licenciada, con mención en psicología clínica, Pontificia Universidad Católica del Perú. Lima: PUCP.

Rabello, L. (2001). Infancia y adolescencia en la cultura del consumo. México: Lumen.

Rodrigo, M.J., Maíquez, M.L.; Martin, J.C.; Byrne, S. (2008). Preservación Familiar: Un enfoque positivo para la intervención con familias. Madrid: Pirámide.

Sáenz, G. (2010). Sintomatología depresiva y acoso escolar en un grupo de adolescentes escolares. Pontificia Universidad Católica del Perú. Lima: PUCP.

Vega, M. y Moro, L. (2013). La representación social de los malos tratos infantiles en la familia: factores psicosociales que influyen en la percepción de las conductas de maltrato. Psychosocial Intervention / Intervención Psicosocial, 22 (1) Abr, 7-14.

Fecha de recepción: 31 de octubre de 2014

Fecha de aceptación: 12 de noviembre de 2014 